\title{
MRI Pattern of Various Cochlear Implant Electrodes In Vivo
}

\author{
Holger Sudhoff ${ }^{1}$ Conrad Riemann ${ }^{1,2}$ Hans Björn Gehl ${ }^{1}$ Ingo Todt ${ }^{1}$
}

\author{
${ }^{1}$ Department of Otolaryngology, Head and Neck Surgery, \\ Bielefeld University, Campus Mitte, Germany \\ ${ }^{2}$ Department of Radiology, Klinikum Bielefeld, Germany
}

Address for correspondence Ingo Todt, Dr. Med., Department of Otolaryngology, Head and Neck Surgery, Bielefeld University, Teutoburgerstr.50, 33604 Bielefeld, Germany (e-mail: todt@gmx.net).

Ann Otol Neurotol:2019;2:51-55

\begin{abstract}
Keywords

- magnetic resonance imaging

- cochlear implant

- electrode
\end{abstract}

Introduction Recent developments regarding cochlear implant magnets (e.g., a bipolar diametral magnet) and refined surgical technique (e.g., implant positioning) have made a significant impact on the relation between a cochlear implant and magnetic resonance imaging (MRI). MRI scanning has changed from a contraindication to a diagnostic tool. For the first time, a pain-free in vivo evaluation of the cochlea's fluid state, following the insertion of an electrode, has become possible via MRI scanning. The aim of this study was to evaluate various cochlear implant electrodes' MRIspecific patterns.

Materials and Methods In a retrospective study, we evaluated the MRI pattern of casting iron (CI) electrodes in a 3T T2 turbo spin echo (TSE) sequence after a surgery with Medel Flex 28, Flex 24, AB HFMS, and MRI at 1.5 T T2 TSE with the Oticon EVO array. Results A generally different axial MRI pattern between the "lateral wall" electrodes and the "modiolar" electrodes at the basal turn could be observed. A difference in terms of electrode length/insertion depth could not be found at the present scan resolution. Electrode contacts have an impact on the MRI signal-diminishing pattern. Conclusion At T2 sequences, an electrode design-specific MRI pattern can be observed.

\section{Introduction}

The intracochlear electrode position after a cast iron $(\mathrm{CI})$ electrode insertion is of central important for the audiological outcome. ${ }^{1}$ Radiological techniques associated with ionized radiation (computed tomography [CT], digital volume tomography [DVT], flat panel tomography [FPT]) can evaluate this important question for surgeons, technicians, and audiologists. Radiation-free options were recently introduced for the positional estimations of the electrode position. Intraoperative electrophysiological measurements ${ }^{2}$ allow estimation, with limitations in terms of the electrodes used and brand-specific software limitations.

An additional way is the use of magnetic resonance imaging (MRI) scans to observe this important question. Unfortunately, during MRI scanning complications such as pain or magnet dislocation ${ }^{3.4}$ can occur at 1.5 T. For some devices, MRIs cannot be performed without the removal of the magnet at $3 \mathrm{~T}$ (6er implant series; Cochlear Company, Sydney, Australia). Therefore, MRI scans are associated with restrictions.

Recently, it was shown that the positioning of the cochlear implant magnet is of central importance for an MRI-based, reproducible visualization of the internal auditory canal and the cochlea. ${ }^{5,6}$ Another study showed that an evaluation of the electrode position at $1.5 \mathrm{~T}$ for modiolar electrodes can be performed. ${ }^{7}$

Specific magnet designs (diametrically bipolar) offer the opportunity for 3T MRI scans without any complications. ${ }^{8}$ Scans of a field strength of $3 \mathrm{~T}$ are known for an increased visual resolution. Beside the experiences with modiolar 
electrodes, ${ }^{7}$ even with lateral wall electrodes an estimation of the scalar electrode position is possible. But the estimation for the latteral wall electrode design is limited to the basal turn. ${ }^{9}$

The aim of this study was related to the variance of electrode designs to evaluate electrode-specific MRI characteristics.

\section{Materials and Methods}

The study was approved by the Institutional Review Board of the Klinikum Bielefeld, Germany (IRB-klibi-HNO-2019/01). Patients provided their written informed consent for the use of their clinical records in this retrospective study.

This retrospective chart review included all patients who underwent MRI scanning at 1.5 T or 3 T between 2014 and 2019. Reasons for MRI scanning were vertigo, headache, and loss of performance. ${ }^{10}$ In terms of electrodes, 22 Flex 28 (Medel; Austria, Innsbruck), 3 Flex 24, 3 High Focus MidScala (HFMS) 3D (Advanced Bionics; Stäfa, Swiss), and 3 EVO (Oticon; Valauris, France) were observed.

In all cases, the implant position/magnet position was intraoperatively determined and positioned 7 to $9 \mathrm{~cm}$ behind the external auditory canal.

All examinations were performed in a $1.5-\mathrm{T}$ or 3-T MR imaging unit (Achieva; Philips Medical Systems, Best, the Netherlands) in coronal and axial plains.

\section{MRI Scanning Parameters}

TSE T2 2D: TR: $3000 \mathrm{~ms}$, TE $120 \mathrm{~ms}$, slice thickness $0.8 \mathrm{~mm}$, voxel size $0.449 \mathrm{~mm}$, F0V $230 \times 199.35$ slices.

\section{Results}

In MRI, scanning was performed without any pain or discomfort to the patient. Looking at the group of Flex 28 patients, the position of the electrode in the lateral part of the scala in the basal turn and axial pattern was easily visualized (-Fig. 1). In the coronal view, a double $\mathrm{C}$ could be seen

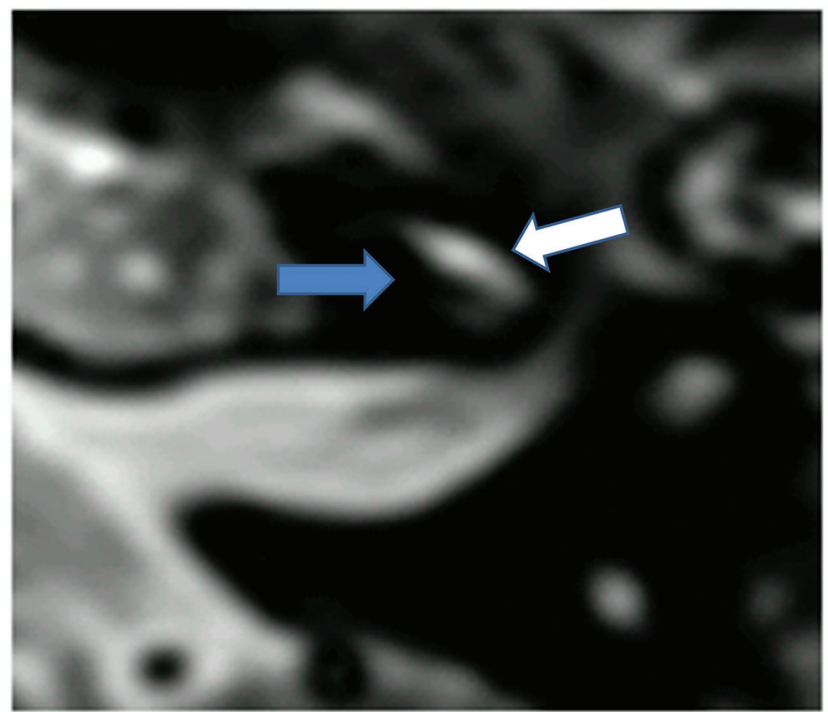

Fig. 1 3T Flex 28 axial. Blue arrow indicates ST diminishing of electrode. White arrow indicates SV. ST, scala tympani; SV, scala vestibuli.
(-Fig. 2). This pattern did not differ from the Flex 24 signal in the axial or even in the coronal view (-Figs. 3 and $\mathbf{4}$ ). The Oticon EVO electrode did not differ in the axial view ( - Fig. 5 ) but showed different signals in the coronal view (-Fig. 6). In the coronal view, the diminishing artifact was larger than the Flex electrodes and most likely caused by the larger bipolar contacts. The HFMS electrode showed a significant difference from the other electrodes in the axial view. Lateral to the electrode, a T2-weighted fluid signal persisted, indicating the electrode's modiolar position ( - Fig. 7 ). In the coronal view, the electrode caused a diminishing signal, which was characterized by a pattern that did not allow for a clear differentiation between the electrode types ( $\mathbf{- F i g . 8}$ ).

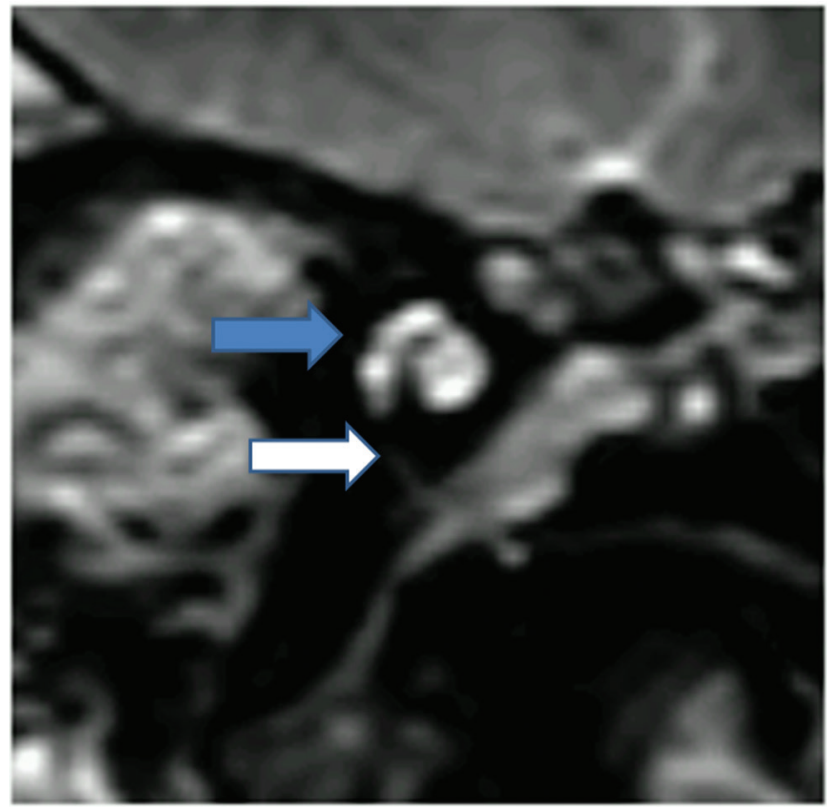

Fig. 2 3T Flex 28 coronal. Blue arrow indicates a decent decrease of fluid signal related to the contact artifact. White arrow indicates intense diminishing by the electrode in the basal turn.

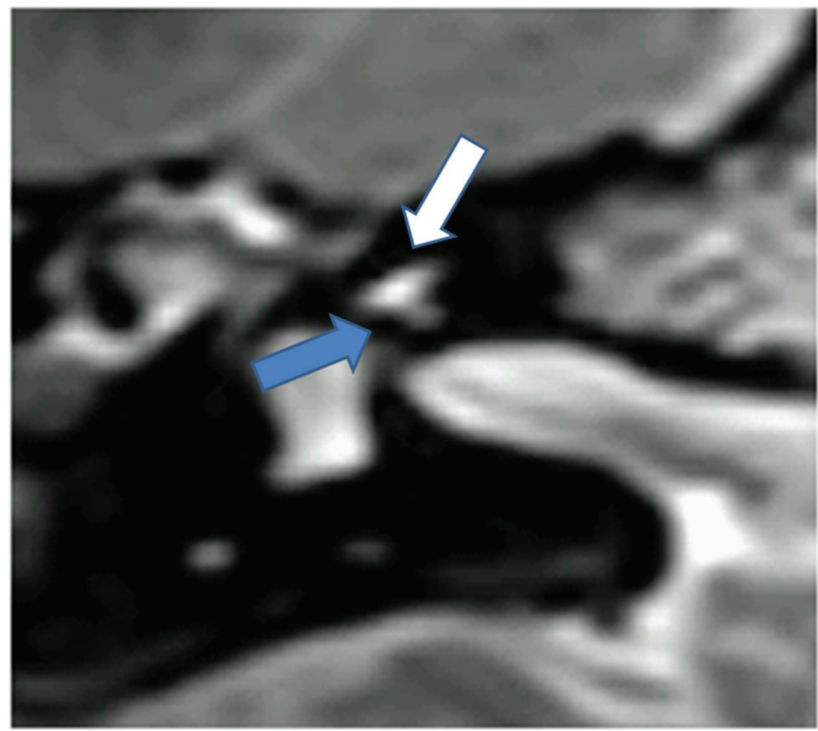

Fig. 3 3T Flex 24 axial. Blue arrow indicates electrode diminishing in the first turn. White arrow indicates SV in the first turn. SV, scala vestibuli. 


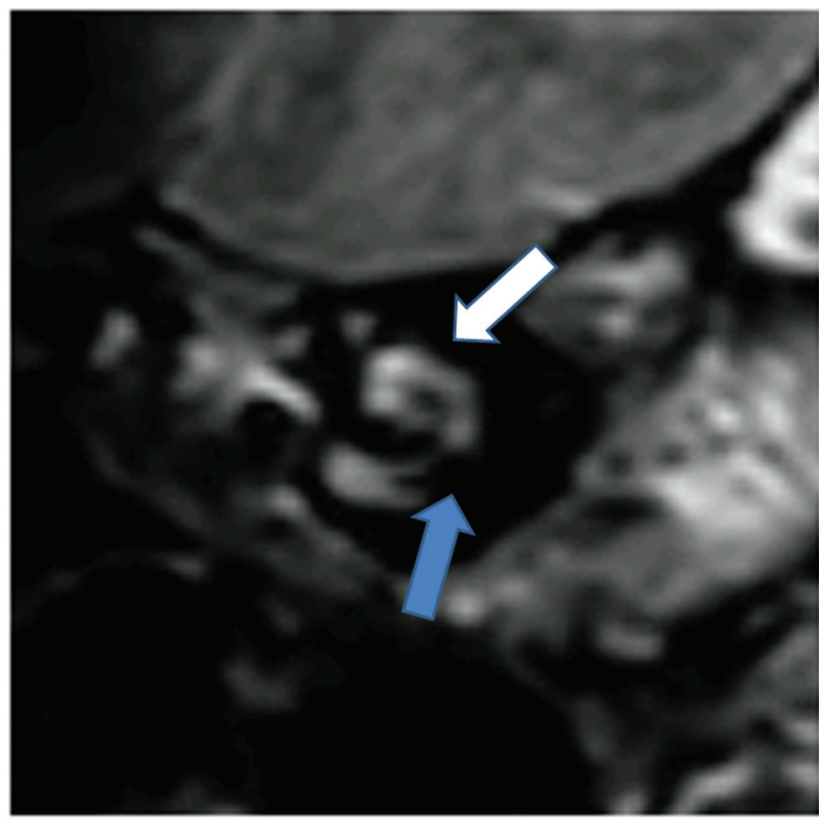

Fig. 4 3T Flex 24 coronal. Blue arrow indicates electrode diminishing in the basal turn. White arrow indicates decent contact artifact.

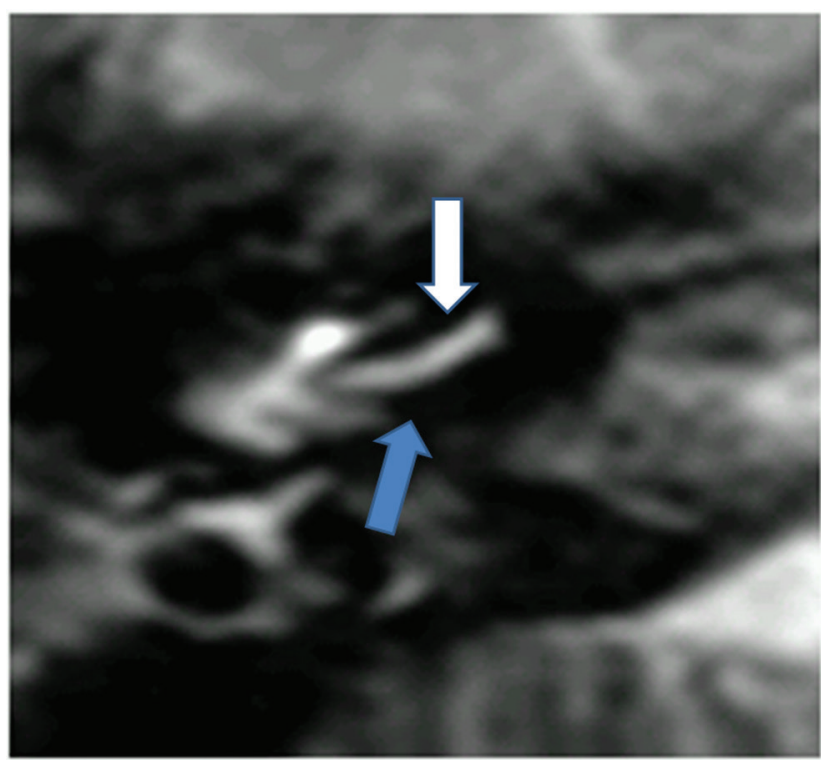

Fig. 5 1.5T EVO axial. Blue arrow indicates electrode diminishing of the ST. White arrow indicates SV. ST, scala tympani; SV, scala vestibuli.

\section{Discussion}

The electrode's scalar position inside the cochlea is of highclinical importance, as it influences the audiological outcome significantly. ${ }^{1,11}$ The estimation of the electrodes' position is a quality control mechanism and is affected by anatomy and electrode design, which evaluate the surgeon's performance.

Until now, radiological techniques have been used for the visual electrode assessment with ionized radiation. An electrophysiological assessment of electrode position seems to be promising but is limited to electrode-dependency. ${ }^{2}$ In addition, software factors and neuronal variations limit the use.

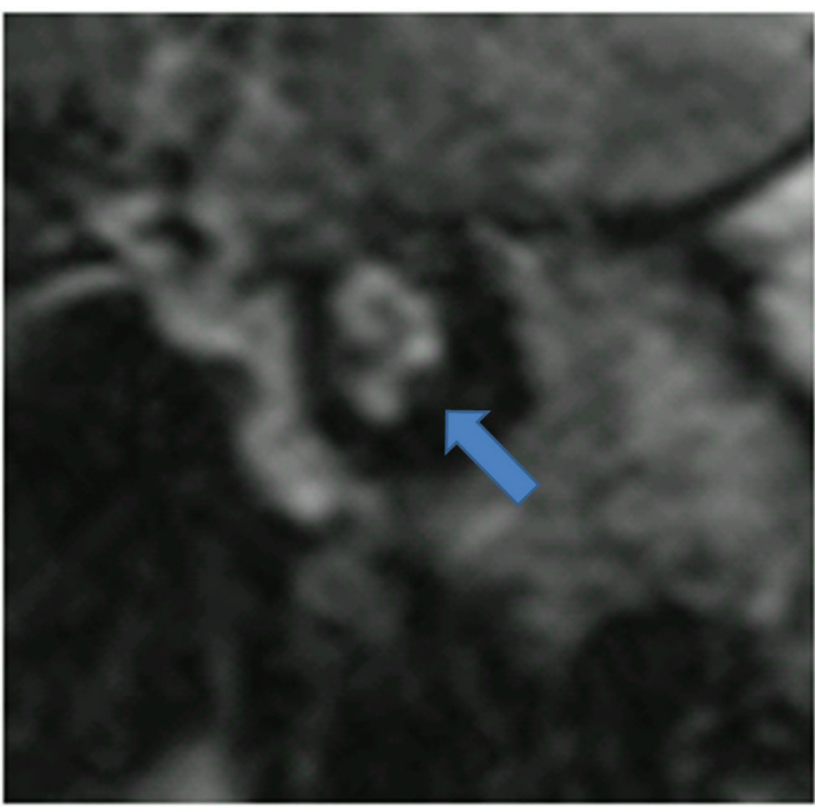

Fig. 6 1.5T EVO coronal. Blue arrow indicates square electrode contact diminishing signal.

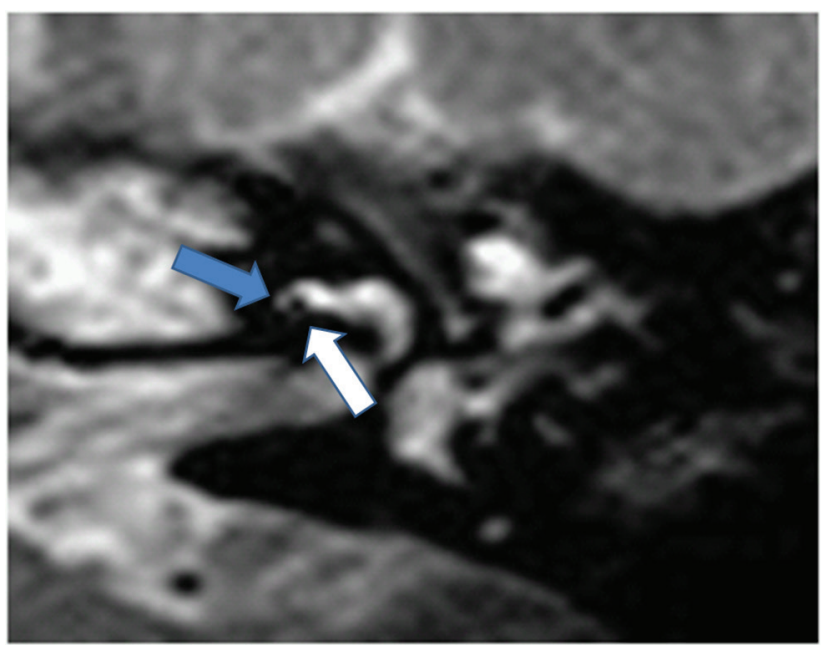

Fig. 7 3T HFMS axial. Blue arrow indicates persisting lateral fluid signal. White arrow indicates electrode-diminishing signal. HFMS, High Focus Mid-Scala.

Although MRI scanning with cochlear implants is possible with headbands at certain field strength, ${ }^{12,13}$ there is a persisting risk of magnet dislocation and pain., ${ }^{3,4}$

New magnet systems such as the diametrically bipolar internal magnet and screw-based implant fixation solved the problem of magnet dislocation and pain. ${ }^{6,14}$ It can be assumed that the new cylindrical 3D bipolar magnet system offers a similar behavior.

Regions of central importance for the otologist-like cochlea and internal auditory canal can be postoperatively visually observed ${ }^{5,6}$ by an implant position-dependent artifact shift.

So far, it has been shown that an estimation of the electrode position by MRI at $1.5 \mathrm{~T}$ is possible. ${ }^{7}$ The electrode 


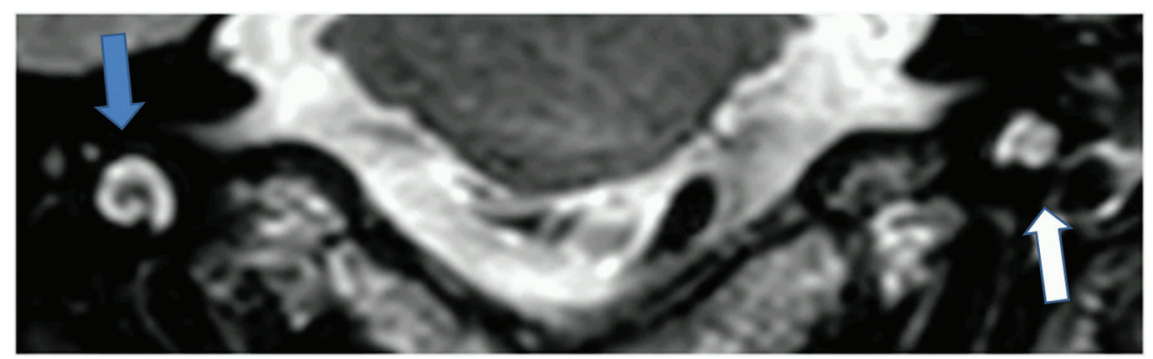

Fig. 8 3T HFMS coronal. Blue arrow indicates no-inserted cochlea. White arrow indicates HFMS-inserted cochlea. HFMS, High Focus Mid-Scala.

position can be estimated even at $3 \mathrm{~T}$ with lateral wall electrodes. ${ }^{9}$ However, in relation to missing scalar dislocations, a clear pattern persists only for the basal turn.

In our study, we retrospectively compared the MRI patterns of various electrode types and designs. We observed no visible difference between a Flex 28 and a Flex 24 electrode, especially in the coronal view, which means that with the currently used $0.8-\mathrm{mm}$ resolution, an insertion-depth difference cannot be observed. As a higher resolution can only be reached by sequences that generate larger artifacts (3D T2, e.g., Drive and CISS), limitations still persist.

Contact artifact effects can be observed for the EVO electrode. Related to the bilateral and in comparison, to the Flex and HFMS electrodes, large contacts of the electrode sharp square diminish visible artifacts, which seem to be characteristic of this electrode. This effect can be observed in the coronal view. The pattern in the axial view is similar to that of the other lateral wall electrodes.

The HFMS electrode's modiolar MRI pattern is characterized by the fluid signal lateral to the electrode in the basal turn. This key finding helps us differentiate between a lateral wall electrode and a modiolar electrode when we assess an MRI scan of an implanted cochlea.

A limitation of the performed study is the scans' resolutions. It can be assumed that with further refined scanning protocols and prolonged scanning times, a better resolution is possible. Currently, the number of slices through the cochlea in the coronal view varies from 3 to 4 and in the axial view from 7 to 8 , with a scanning resolution of $0.8 \mathrm{~mm}$. With an increased resolution, more visual characteristics should be determinable.

If temperature is increased to approximately $43^{\circ} \mathrm{C}$ for longer than 30 minutes, ${ }^{15}$ heating is known to have a negative effect on neural structures. A 3-T MRI scan-induced temperature change is less than $3^{\circ} \mathrm{C}$ for 15 -minute scans. Usually, peaks of temperature increase occur during the first 3 to 5 minutes (personal communication; MEDEL, Innsbruck, Austria). Therefore, even high-energy sequences are, with the current knowledge, without danger.

In relation to the introduction of new magnet systems, MRI scanning is currently changing from a contraindication to a diagnostic tool. Previous limitations, such as demagnetization, pain, and dislocation, no longer persist. Other factors such as scanning time, resolution, and sequences arise. This change offers new chances, especially in terms of electrode assessment.
Ionized free-electrode assessment by MRI allows for scanning in children. Therefore, general knowledge about various electrodes' MRI pattern characteristics is very important.

\section{Conclusion}

At T2 TSE sequences, an electrode design-specific MRI pattern can be observed.

\section{Ethical Approval}

The study was approved by the Institutional Review Board of the Klinikum Bielefeld, Germany (IRB-klibi-HNO2019/01). Patients provided their written informed consent for the use of their clinical records in this study. The study was conducted ethically in accordance with the World Medical Association Declaration of Helsinki.

\section{Note}

Data are available from the corresponding author upon request.

\section{Authors' Contributions}

I. T. contributed to conception of study and writing the manuscript. H.B. G. participated in discussion concerning radiological results and contributed to writing the manuscript. H. S. and C. R. contributed to writing the manuscript.

\section{Funding}

None.

\section{Conflict of Interest}

None declared.

\section{References}

1 Aschendorff A, Kubalek R, Turowski B, et al. Quality control after cochlear implant surgery by means of rotational tomography. Otol Neurotol 2005;26(1):34-37

2 Mittmann P, Ernst A, Todt I. Intraoperative electrophysiologic variations caused by the scalar position of cochlear implant electrodes. Otol Neurotol 2015;36(6):1010-1014

3 Grupe G, Wagner J, Hofmann S, et al. [Prevalence and complications of MRI scans of cochlear implant patients: German version]. HNO 2016;64(3):156-162

4 Hassepass F, Stabenau V, Maier W, et al. Revision surgery due to magnet dislocation in cochlear implant patients: an emerging complication. Otol Neurotol 2014;35(1):29-34

5 Walton J, Donnelly NP, Tam YC, et al. MRI without magnet removal in neurofibromatosis type 2 patients with cochlear and auditory brainstem implants. Otol Neurotol 2014;35(5):821-825 
6 Todt I, Rademacher G, Mittmann P, Wagner J, Mutze S, Ernst A. MRI artifacts and cochlear implant positioning at $3 \mathrm{~T}$ in vivo. Otol Neurotol 2015;36(6):972-976

7 Stratmann A, Mittmann P, Rademacher G, et al. MRI-based estimation of scalar cochlear-implant electrode position. BioMed Res Int 2017;2017:6372704

8 Todt I, Tittel A, Ernst A, Mittmann P, Mutze S. Pain free $3 \mathrm{~T}$ MRI scans in cochlear implantees. Otol Neurotol 2017;38(10):e401-e404

9 Tek F, Müller S, Boga E, et al. 3 T high resolution MRI based estimation of scalar cochlear implant electrode position. Acta Otol Ital 2019;39:1-5

10 Mittmann P, Ernst A, Scholz S, et al. Cochlear implantation for single-sided deafness: observations in poor performers. Annals Otol Neurotol 2018;1(1):18-22

11 Finley CC, Holden TA, Holden LK, et al. Role of electrode placement as a contributor to variability in cochlear implant outcomes. Otol Neurotol 2008;29(7):920-928
12 Kim BG, Kim JW, Park JJ, Kim SH, Kim HN, Choi JY. Adverse events and discomfort during magnetic resonance imaging in cochlear implant recipients. JAMA Otolaryngol Head Neck Surg 2015;141(1):45-52

13 Crane BT, Gottschalk B, Kraut M, Aygun N, Niparko JK. Magnetic resonance imaging at $1.5 \mathrm{~T}$ after cochlear implantation. Otol Neurotol 2010;31(8):1215-1220

14 Todt I, Rademacher G, Grupe G, et al. Cochlear implants and 1.5 T MRI scans: the effect of diametrically bipolar magnets and screw fixation on pain. J Otolaryngol Head Neck Surg 2018;47(1):11

15 Coffey RJ, Kalin R, Olsen JM. Magnetic resonance imaging conditionally safe neurostimulation leads: investigation of the maximum safe lead tip temperature. Neurosurgery 2014;74(2):215-224 